\title{
Hyperdiffusion of quantum waves in random photonic lattices
}

\author{
Alexander Iomin \\ Department of Physics, Technion, Haifa, 32000, Israel \\ Phys. Rev. E 92, 022139 (2015)
}

\begin{abstract}
A quantum-mechanical analysis of hyper-fast (faster than ballistic) diffusion of a quantum wave packet in random optical lattices is presented. The main motivation of the presented analysis is experimental demonstrations of hyper-diffusive spreading of a wave packet in random photonic lattices [L. Levi et al., Nature Phys. 8, 912 (2012)]. A rigorous quantum-mechanical calculation of the mean probability amplitude is suggested, and it is shown that the power law spreading of the mean squared displacement (MSD) is $\left\langle x^{2}(t)\right\rangle \sim t^{\alpha}$, where $2<\alpha \leq 3$. The values of the transport exponent $\alpha$ depend on the correlation properties of the random potential $V(x, t)$, which describes random inhomogeneities of the medium. In particular, when the random potential is $\delta$ correlated in time, the quantum wave packet spreads according Richardson turbulent diffusion with the MSD $\sim t^{3}$. Hyper-diffusion with $\alpha=12 / 5$ is also obtained for arbitrary correlation properties of the random potential.
\end{abstract}

PACS numbers: 42.25.Dd, 05.40.-a, 03.65.-w

\section{INTRODUCTION}

Recently, it has been demonstrated, experimentally and numerically [1] that space-time disordered media accelerate the transport in a way, when an initial wave packet spreads at a rate faster than ballistic with the mean squared displacement (MSD) $\left\langle x^{2}(t)\right\rangle \sim t^{12 / 5}$. This effect has been explained in the framework of classical mechanical approach due to continuous expansion of the transverse momentum spectrum in an arbitrary spacetime random potential [1-3]. In this paper we suggest a quantum-mechanical explanation of this experimental observation of the disorder enhanced transport in photonic lattices [1], which is a more general approach for a quantum wave packet spreading in randomly inhomogeneous media [1, 4, 5].

An investigation of wave spreading in randomly inhomogeneous media is a long lasting problem, which has been well reviewed already more than thirty years ago [6, 7], where a variety of applications have been considered, and this theory has also a strong impact on statistical methods in physics [8] (see also recent review [9]).

The main objective of the present research is an estimation of the mean squared displacement (MSD) of the wave packet spreading in the transversal direction (which is the $x$ axis) under its propagation along a wave-guide. Here the main accent is made on the rigorous calculation of the mean probability amplitude. It is known that a wave propagation with the wavelength $\lambda$ in a long rangedependent wave-guide can be described by the parabolic equation in the limit of a small-angle propagation [6, 10]. This equation corresponds formally to the Schrödinger equation with an effective Planck constant of the order of $\lambda$. Formally, the longitudinal coordinate plays a role of an effective time $t$, and the dynamics takes place in a random potential $V(x, t)$, which is a space-time dependent noise. A rigorous quantum-mechanical consideration is suggested for this Langevin-Schrödinger equa- tion, and the wave function is obtained as functional of $V(x, t)$. We show that the quantum process of spreading depends on the time correlation properties of the random potential. We obtain the hyperfast spreading of the quantum wave packet with the $\operatorname{MSD}\left\langle x^{2}(t)\right\rangle \sim t^{\alpha}$ with the transport exponent $2<\alpha \leq 3$. When the noise is a Markov ( $\delta$-correlated) process, the quantum wave packet dynamics corresponds to Richardson diffusion [11] with the MSD of the order of $t^{3}$. This classical turbulent diffusion is obtained here by the rigorous quantum mechanical treatment. A phenomenological statistical approach dated back to works by Kolmogorov and Obukhov [12, 13] suggested this turbulent acceleration by means of a Gaussian $\delta$-correlated noise 14], added to the dynamical system $\ddot{x}+V(t)=0$. In this case, due to the noise term $V(t)$, Richardson diffusion takes place with the $\operatorname{MSD}\left\langle x^{2}(t)\right\rangle \sim t^{3}$, which is due to the diffusive spread of the velocity profile $\left\langle\dot{x}^{2}(t)\right\rangle \sim t$. In quantum mechanics, the Kolmogorov-Obukhov approach was first applied in Ref. [15] to study a non-diffusive motion, where a Gaussian $\delta$ correlated in time random process was treated in the framework of the Furutsu-Novikov formula [16, 17] for the mean probability amplitudes [6, 9]. Recently, it was applied to described a tracer behavior [18] for an explanation of a limiting case of experimental realization of quantum-mechanical superdiffusion of ultra cold atoms [19].

However, in real experimental realizations of the disorder in photonic lattices [4, 5], the random potential does not possess this Markov property due to the finite size of the optical wavelength $\lambda$. Therefore, the quantum dynamics is considered in an arbitrary random potential $V(x, t)$, which is correlated in both time and space. In this case a rigorous quantum-mechanical analysis cannot be performed, and a suitable approximation is suggested to treat this random quantum dynamics.

It is well known that the quantum dynamics can be 
described by a complex Gaussian kernel ${ }^{1}$ in functional integration [20, 21]. When $V(x, t)$ is $\delta$ correlated in time, it does not affect the quantum Gaussian paths in the functional integration that makes it possible to treat the quantum mechanics rigorously, like in the Richardson diffusion case. The situation changes essentially, when the random process is strongly correlated. Then the quantum paths are affected by the inhomogeneities of the media. The rigorous analysis is impossible, and terms, which are responsible for this "intertwine", are treated approximately by averaging this part of the quantum paths. The suggested averaging procedure is performed in self-consistency with the quantum spreading, and as a result of this, we obtain hyperdiffusion of the quantum packet spreading, when the MSD is of the order of $t^{12 / 5}$. which coincides with a result obtained in Refs. [2, 3] in the ray dynamics limit.

Therefore, as the result of the parabolic equation approximation of the wave process, the wave spreading in randomly inhomogeneous media is investigated in the framework of quantum mechanics with a random potential, which is the Langevin Schrödinger equation. An important motivation for this analysis is experimental investigations of quantum wave packet spreading in random optical lattices [1, 4, 5]. Another interesting motivation of the present analysis is investigation (experimental and theoretical) of sound waves spreading in underwater acoustics in the presence of random environments (see e.g. recent results in Refs. [22, 23] ).

\section{A. Parabolic equation approximation}

The method of parabolic equation approximation was first applied by Leontovich in studying radio-waves spreading 24] and later it has been developed in detail by Khohlov [25] (see also [10]). Parabolic equation for monochromatic light propagation in two dimensional randomly inhomogeneous media reads [1, 4, 6]

$$
i \partial_{z} \Psi=\left[-\frac{1}{2 k} \partial_{x}^{2} \Psi-\frac{k}{n_{0}} \Delta n(z, x)\right] \Psi .
$$

Here $\Delta n(x, z)$ is local fluctuations of refractive index $n=n_{0}+\Delta n$, and $z$ is the propagation direction of the wave with the wave index $k=2 \pi n_{0} / \lambda$, therefore an effective semiclassical parameter is of the order of $1 / k$. In what follows it is convenient to work with dimensionless variables and parameters. Taking into account that Eq. (11) has a form of a Schrödinger equation, one defines the dimensionless effective time $t=z / \lambda$ and the dimensionless effective Planck constant $\tilde{h}=\frac{1}{k \lambda}=\frac{1}{2 \pi n_{0}}$, then, the

\footnotetext{
1 This presentation of the quantum dynamics by means of an auxiliary Markov field in the framework of the Feynman-Kac formula does not suppose any Markovian property of quantum mechanics.
}

dimensionless quantum momentum is $\frac{\lambda}{k \lambda} \partial_{x}=\tilde{h} \partial_{x}$, where $x / \lambda \rightarrow x$. Note that the wavelength in the experimental setup is $\lambda \sim 0.514 \mu \mathrm{m}$ and $\Delta n / n_{0} \sim 10^{-4} \ll 1$ [1]. Therefore, the effective Planck constant is a small semiclassical parameter.

\section{QUANTUM LANGEVIN EQUATION}

Formally, the wave function $\Psi(x, t)$ describes the dynamics of a quantum wave packet (particle) in random time-dependent optical potential $V(x, t)$, and it is governed by the Schrödinger equation, which reads

$$
\partial_{t} \Psi(x, t)=\left[i \tilde{h} \partial_{x}^{2} / 2+i V(x, t) / \tilde{h}\right] \Psi(x, t)
$$

with the initial condition

$$
\Psi(x, t=0)=\Psi_{0}(x) .
$$

Considering the optical random potential $V(x, t)$ as an expansion of a quasiperiodic function, one has [1, 3]

$$
V(x, t)=\frac{1}{\sqrt{N}} \sum_{m=-N}^{N} A_{m} \exp \left(i k_{m} x-i \omega_{m} t\right)+c . c .,
$$

where the coefficients of the expansion $A_{m}$ are random complex values, while $k_{m}$ and $\omega_{m}$ are independent random real values. Denoting averaging over the Gaussian ensemble by $\langle\ldots\rangle_{V}$, we obtain that $A_{m}$ are controlled by a Gaussian distribution with the averaging property

$$
\left\langle A_{m}\right\rangle_{V}=\left\langle A_{m} A_{n}\right\rangle_{V}=0, \quad\left\langle A_{m}^{*} A_{n}\right\rangle_{V}=\sigma^{2} \delta_{m, n} .
$$

From this property, one obtains for the 1D space-time dependent potential $V(x, t)$

$$
\left\langle|V(x . t)|^{2}\right\rangle_{V}=2 \sigma^{2} .
$$

Note that this formulation of the random potential is general and corresponds to the experimental setup [1]. Since $V(x, t)$ is a random function, the Schrödinger Eq. (2) is a Langevin equation with a multiplicative noise potential $V(x, t)$.

Following Ref. [6], this equation can be solve exactly. The solution of Eq. (2) can be presented in the form of a functional integration over an auxiliary Gaussian field $\lambda(t)$. The details of the calculation can be found in Ref. [9]. However, here we present an alternating way of the solution, which is more suitable for the quantummechanical consideration.

\section{A. Solution of Langevin equation}

A formal integration of the Schrödinger Eq. (2) yields a $T$ ordered (time ordered) form of the evolution operator, which acts on the initial wave function

$$
\Psi(x, t)=\hat{T} \exp \left[\frac{i \tilde{h}}{2} \int_{0}^{t} \partial_{x}^{2} d \tau+\frac{i}{\tilde{h}} \int_{0}^{t} V(x, \tau) d \tau\right] .
$$


Under the sign of the time ordering operator $\hat{T}$, all values are commuted, and the kinetic and potential exponentials can stay separate. Therefore, for the kinetic term, one applies the Hubbard-Sratonovich transformation [26, 27]

$$
\begin{gathered}
\exp \left[\frac{i \tilde{h}}{2} \int_{0}^{t} \partial_{x}^{2} d \tau\right]=\int \prod_{\tau} \frac{d \lambda(\tau)}{\sqrt{2 \pi \tilde{h} i}} \\
\times \exp \left[\frac{i}{2 \tilde{h}} \int_{0}^{t} d \tau \lambda^{2}(\tau)\right] \cdot \exp \left[\int_{0}^{t} d \tau \lambda(\tau) \partial_{x}\right] .
\end{gathered}
$$

Taking into account that the last exponential acts as a shift operator, one obtains the solution

$$
\begin{gathered}
\Psi(x, t)=\int \prod_{\tau} \frac{d \lambda(\tau)}{\sqrt{2 \pi \tilde{h} i}} \exp \left[\frac{i}{2 \tilde{h}} \int_{0}^{t} d \tau \lambda^{2}(\tau)\right] \\
\times \Psi_{0}\left(x+\int_{0}^{t} d \tau \lambda\right) \exp \left[\frac{i}{\tilde{h}} \int_{0}^{t} d \tau V\left(x+\int_{\tau}^{t} d \tau^{\prime} \lambda, \tau\right)\right] .
\end{gathered}
$$

Therefore, the quantum-mechanical estimation of the MSD $\left\langle x^{2}(t)\right\rangle$ leads to two standard procedures of averaging. First one obtains a mean probability amplitude ${ }^{2}$ $\left\langle|\Psi(x, t)|^{2}\right\rangle_{V}$ by averaging of the obtained result in Eq. (9) over all realizations of the random field $V(x, t)$, and then performs a standard quantum-mechanical calculation of the MSD. Therefore the MSD reads

$$
\left\langle x^{2}(t)\right\rangle=\int x^{2}\langle| \Psi\left(x,\left.t\right|^{2}\right\rangle_{V} d x
$$

\section{MEAN PROBABILITY AMPLITUDE}

For the random quantum process, the physical characteristics are described by the mean probability amplitude (MPA), or distribution function $\rho(x, t)$, obtained from the random wave function (9) by averaging over the Gaussian distribution

$$
\rho(x, t)=\left\langle|\Psi(x, t)|^{2}\right\rangle_{V}
$$

Obviously, this value is normalized $\int d x \rho(x, t)=1$. Following [28 30], let us obtain this normalization condition. The initial condition can be presented by means of the Fourier integration

$$
\Psi_{0}(x)=\frac{1}{2 \pi} \int_{-\infty}^{\infty} \bar{\Psi}_{0}(k) e^{-i p x} d p
$$

\footnotetext{
2 Note that an important information about the random process is carried by the correlation function of $V(x, t)$, which can be obtained by the ensemble averaging.
}

Substituting this expression in Eq. (9), one obtains for the MPA

$$
\begin{aligned}
\rho(x, t) & =\int \prod_{\tau} \frac{d \lambda_{1}(\tau) d \lambda_{2}(\tau)}{2 \pi \tilde{h}} \exp \left[\frac{i}{2 \tilde{h}} \int_{0}^{t}\left(\lambda_{1}^{2}-\lambda_{2}^{2}\right) d \tau\right] \\
& \times \int \frac{d p_{1} d p_{2}}{4 \pi^{2}} \bar{\Psi}_{0}\left(p_{1}\right) \bar{\Psi}_{0}^{*}\left(p_{2}\right) \exp \left[-i x\left(p_{1}-p_{2}\right)\right] \\
& \times \exp \left\{-i \int_{0}^{t}\left[p_{1} \lambda_{1}(\tau)-p_{2} \lambda_{2}(\tau)\right] d \tau\right\} \\
& \times\left\langle\exp \left[\frac{i}{\tilde{h}} \int_{0}^{t}\left(V\left(\tilde{x}_{1}, \tau\right)-V^{*}\left(\tilde{x}_{2}, \tau\right)\right) d \tau\right]\right\rangle_{V},
\end{aligned}
$$

where $\tilde{x}_{j}=x+\int_{\tau}^{t} \lambda_{j}\left(\tau^{\prime}\right) d \tau^{\prime}$ and $j=1,2$.

\section{A. Integration over the Gaussian distribution}

Now one can treat the random potential term by integration over the $2 N+1$ dimensional Gaussian packet, and this procedure coincides with integration over manydimensional coherent states [31]

$$
d\left[\mathcal{P}\left(\left\{A_{m}^{*}, A_{m}\right\}\right)\right]=\exp \left(-\sum_{m}\left|A_{m}\right|^{2} / \sigma^{2}\right) \prod_{m} \frac{d^{2} A_{m}}{\pi \sigma^{2}},
$$

where $d^{2} A_{m}=d\left[\operatorname{Re}\left(A_{m}\right)\right] d\left[\operatorname{Im}\left(A_{m}\right)\right]$. Therefore, after taking into account Eq. (4), the ensemble averaging procedure corresponds to the following $2 N+1$ dimensional integration

$$
\begin{aligned}
\langle\ldots\rangle_{V} & =\prod_{m} \int \frac{d^{2} A_{m}}{\pi \sigma^{2}} \exp \left[-\sum\left|A_{m}\right|^{2} / \sigma^{2}\right] \\
& \times \exp \left[\sum_{m}\left(A_{m} \alpha_{m}^{*}-A_{m}^{*} \alpha_{m}\right)\right],
\end{aligned}
$$

where $\alpha_{m}$ is the following complex function

$\alpha_{m}=\frac{i}{\tilde{h} \sqrt{N}} \int_{0}^{t} d \tau\left[e^{-i k_{m} \tilde{x}_{1}(\tau)+i \omega_{m} \tau}-e^{-i k_{m} \tilde{x}_{2}(\tau)+i \omega_{m} \tau}\right]$.

Using the property of integration of coherent states [31], namely

$$
\int \frac{d^{2} \beta}{\pi} e^{-|\beta|^{2}} e^{\alpha^{*} \beta} f\left(\beta^{*}\right)=f\left(\alpha^{*}\right),
$$

one obtains from the integration in Eq. (15)

$$
\langle\ldots\rangle_{V}=\exp \left[-\sigma^{2} \sum_{m}\left|\alpha_{m}\right|^{2}\right] \equiv \mathcal{F}\left[\lambda_{1}(\tau), \lambda_{2}(\tau)\right] .
$$

The next step of the quantum analysis is functional integration over the auxiliary Gaussian fields $\lambda_{1}$ and $\lambda_{2}$. However, the exact quantum-mechanical treatment is 
possible only for the $\delta$ correlated in time random potential $V(x, t)$

$$
\left\langle V^{*}(x, t) V\left(x^{\prime}, t^{\prime}\right)\right\rangle_{V}=C\left(x, t ; x^{\prime}, t^{\prime}\right)=C\left(x, x^{\prime}\right) \delta\left(t-t^{\prime}\right),
$$

where $C(x, x)=2 \sigma^{2}$ (cf. Eq. (6) $)$. First, we consider this case, noting that the restriction of $\delta$ correlation corresponds also to the Obukhov mechanism of Richardson diffusion [14].

\section{RICHARDSON DIFFUSION}

Richardson diffusion [11] was the first phenomenological observation of developed turbulence [32], and this phenomenon has been discussed in a variety of experimental and numerical studies, see reviews [12, 32] and as admitted in [32, 33], it still lacks sufficient experimental confidence.

Let us define the property of $V(x, t)$ by means of the spectral density $S(k, \omega)$ of the correlation function $C\left(x, t ; x^{\prime}, t^{\prime}\right)$ with the $\delta$ correlated constraint (19). Following Refs. [2, 3], we present the correlation function in the following translational invariant in space and time form

$$
\begin{aligned}
& C\left(x, x^{\prime}\right) \delta\left(t-t^{\prime}\right)=\frac{\sigma^{2}}{N} \sum_{m}\left[e^{i k_{m}\left(x-x^{\prime}\right)-i \omega_{m}\left(t-t^{\prime}\right)}+c . c .\right] \\
= & \sigma^{2} \int d k \int d \omega \tilde{S}(k, \omega)\left[e^{i\left[k\left(x-x^{\prime}\right)-\omega\left(t-t^{\prime}\right)\right]}+c . c .\right] \\
= & \sigma^{2} \int d k S(k) \cos \left[k\left(x-x^{\prime}\right)\right] \delta\left(t-t^{\prime}\right),
\end{aligned}
$$

where $S(k)=4 \pi \tilde{S}(k, \omega)$.

Using this delta correlated property, one can describe the dynamics of $\left|\alpha_{m}(t)\right|^{2}$ in Eq. (18) by means of the spectral density $S(k)$. Substituting Eq. (16) in Eq. (18) and taking into account Eq. (20), one obtains

$$
\begin{aligned}
& \mathcal{F}\left[\lambda_{1}(\tau), \lambda_{2}(\tau)\right]=\exp \left[-\sigma^{2} \sum_{m}\left|\alpha_{m}\right|^{2}\right] \\
= & \exp \left\{-\frac{\sigma^{2}}{\tilde{h}^{2} N} \int_{0}^{t} d \tau_{1} \int_{0}^{t} d \tau_{2} \sum_{m=-N}^{N}\right. \\
\times & {\left[e^{i k_{m} \tilde{x}_{1}\left(\tau_{1}\right)-i \omega_{m} \tau_{1}}-e^{i k_{m} \tilde{x}_{2}\left(\tau_{1}\right)-i \omega_{m} \tau_{1}}\right] } \\
\times & {\left.\left[e^{-i k_{m} \tilde{x}_{1}\left(\tau_{2}\right)+i \omega_{m} \tau_{2}}-e^{-i k_{m} \tilde{x}_{2}\left(\tau_{2}\right)+i \omega_{m} \tau_{2}}\right]\right\} } \\
= & \exp \left\{-\frac{\sigma^{2}}{\tilde{h}^{2}} \int_{0}^{t} d \tau \int_{-\infty}^{\infty} d k S(k)\right. \\
\times & \left.\left\{1-\cos \left[k \int_{\tau}^{t}\left(\lambda_{1}\left(\tau^{\prime}\right)-\lambda_{2}\left(\tau^{\prime}\right)\right) d \tau^{\prime}\right]\right\}\right\} .
\end{aligned}
$$

To take the functional integrals over the auxiliary fields $\lambda_{1}(\tau)$ and $\lambda_{2}(\tau)$, one performs the following linear change of the fields [28]

$$
\begin{aligned}
& \lambda_{1}(\tau)=2 \mu(\tau)+\tilde{h} \nu(\tau) / 2 \\
& \lambda_{2}(\tau)=2 \mu(\tau)-\tilde{h} \nu(\tau) / 2
\end{aligned}
$$

where the Jakobian of the transformation is $\tilde{h}$ for each value of $\tau$. Then the functional part of the integrand in Eq. (13) reads

$$
\begin{aligned}
& \prod_{\tau} \frac{d \mu(\tau) d \nu(\tau)}{2 \pi} \exp \left[i \int_{0}^{t} \mu(\tau) \nu(\tau) d \tau\right] \\
\times & \exp \left[-2 i\left(p_{1}-p_{2}\right) \int_{0}^{t} \mu(\tau) d \tau\right. \\
- & \left.\frac{i \tilde{h}}{4}\left(p_{1}+p_{2}\right) \int_{0}^{t} \nu(\tau) d \tau\right] \mathcal{F}[\nu(\tau)],
\end{aligned}
$$

where we use the fact that $\mathcal{F}\left[\lambda_{1}(\tau), \lambda_{2}(\tau)\right]=\mathcal{F}\left[\lambda_{1}(\tau)-\right.$ $\lambda_{2}(\tau)$, which follows from Eq. (21). Taking integration over $x$ in Eq. (13) one obtains the $\delta$ function $\delta\left(p_{1}-p_{2}\right)$. Then, functional integration over $\mu(\tau)$ yields the delta functions $\prod_{\tau} \delta(\nu(\tau))$, since the rest of the integrand does not depend on $\mu(\tau)$. Finally, after integration over $\nu(\tau)$ one obtains that the MPA is normalized to 1

$$
\int_{-\infty}^{\infty} \rho(x, t) d x=1 .
$$

\section{A. Mean squared displacement}

Handling the exact expression of the MPA, we arrive at the main objective of the work and can evaluate the rate of the wave packet spreading by calculation of the $\operatorname{MSD}\left\langle x^{2}(t)\right\rangle$ in the (transversal) $x$ direction. Taking into account Eqs. (13) and (21), one obtains for the MSD

$$
\begin{aligned}
& \left\langle x^{2}(t)\right\rangle=\int_{-\infty}^{\infty} \rho(x, t) x^{2} d x \\
= & \int \frac{d p_{1} d p_{2}}{4 \pi^{2}} \bar{\Psi}_{0}\left(p_{1}\right) \bar{\Psi}_{0}^{*}\left(p_{2}\right) \delta^{(2)}\left(p_{1}-p_{2}\right) \\
\times & \int \prod_{\tau} \frac{d \mu(\tau) d \nu(\tau)}{2 \pi} \exp \left[i \int_{0}^{t} \mu(\tau) \nu(\tau) d \tau\right] \\
\times & \exp \left[-2 i\left(p_{1}-p_{2}\right) \int_{0}^{t} \mu(\tau) d \tau\right. \\
- & \left.\frac{i \tilde{h}}{4}\left(p_{1}+p_{2}\right) \int_{0}^{t} \nu(\tau) d \tau\right] \mathcal{F}[\nu(\tau)]
\end{aligned}
$$

where we use the following definition of the second derivative of the delta function $\delta^{(2)}\left(p_{1}-p_{2}\right) \equiv \partial_{p_{1}} \partial_{p_{2}} \delta\left(p_{1}-p_{2}\right)$. Now, we can repeat the previous calculations of Eqs. (13), (21), and (23). Functional integration over $\mu(\tau)$ yields $\prod_{\tau} \delta\left[\nu(\tau)-2\left(p_{1}-p_{2}\right)\right]$. Therefore, functional integration over $\nu(\tau)$ is rigorous, as well. Performing integration with $\delta^{(2)}\left(p_{1}-p_{2}\right)$, one obtains finally for the MSD

$$
\left\langle x^{2}(t)\right\rangle=P_{0}^{2} t^{2}+\frac{D_{0}}{3} t^{3}
$$

Here the first term $\sim t^{2}$ describes a well known wave packet spreading in homogeneous media with the mean 
squared momentum

$$
P_{0}^{2}=\frac{\tilde{h}^{2}}{2 \pi} \int_{-\infty}^{\infty} p^{2}\left|\hat{\Psi}_{0}(p)\right|^{2} d p
$$

The second term, which is obtained by the rigorous quantum mechanical calculations, is of a pure classical nature and corresponds to Richardson diffusion [11]. However, its contribution in the quantum process of the wave packet spreading is dominant $\sim D_{0} t^{3}$, where the generalized diffusion coefficient is

$$
D_{0}=\sigma^{2} \int_{-\infty}^{\infty} k^{2} S(k) d k
$$

\section{HYPER-DIFFUSION}

It should be stressed that the experimental realization of photonic lattices with the $\delta$-correlated random potential is technically impossible [1, 4, 5]. Therefore, the estimation of the MSD for the realistic arbitrary correlated random potential $V(x, t)$ leads to essential complication of the analysis. Let us return to Eqs. (20) and (21) in a general form of the spectral density $S(k, \omega)$. The correlation function reads

$$
\begin{aligned}
& C\left(x-x^{\prime} ; t-t^{\prime}\right)=\frac{\sigma^{2}}{N} \sum_{m}\left[e^{i k_{m}\left(x-x^{\prime}\right)-i \omega_{m}\left(t-t^{\prime}\right)}+c . c .\right] \\
= & \sigma^{2} \int d k \int d \omega \tilde{S}(k, \omega)\left[e^{i\left[k\left(x-x^{\prime}\right)-\omega\left(t-t^{\prime}\right)\right]}+c . c .\right] .
\end{aligned}
$$

In this case, the functional action $\mathcal{I}\left[\lambda_{1}(\tau), \lambda_{2}(\tau)\right]=$ $-\sigma^{2} \sum_{m}\left|\alpha_{m}\right|^{2}$ in

$$
\mathcal{F}\left[\lambda_{1}(\tau), \lambda_{2}(\tau)\right]=\exp \left\{\mathcal{I}\left[\lambda_{1}(\tau), \lambda_{2}(\tau)\right]\right\}
$$

in Eq. (21) is a more complicated expression, which is not treatable rigorously. After some algebraic manipulations, this reads

$$
\begin{aligned}
& \mathcal{I}\left[\lambda_{1}(\tau), \lambda_{2}(\tau)\right]=-\frac{\sigma^{2}}{\tilde{h}^{2}} \int_{0}^{t} d \tau_{1} \int_{0}^{t} d \tau_{2} \int_{-\infty}^{\infty} d k \int_{-\infty}^{\infty} d \omega \\
& \times S(k, \omega)\left[e^{i k \int_{\tau_{2}}^{\tau_{1}} \lambda_{1} d \tau^{\prime}-\omega\left(\tau_{1}-\tau_{2}\right)}\left(1-e^{i k \int_{\tau_{1}}^{t}\left(\lambda_{1}-\lambda_{2}\right) d \tau^{\prime}}\right)\right. \\
& \left.+e^{i k \int_{\tau_{2}}^{\tau_{1}} \lambda_{2} d \tau^{\prime}-\omega\left(\tau_{1}-\tau_{2}\right)}\left(1-e^{-i k \int_{\tau_{1}}^{t}\left(\lambda_{1}-\lambda_{2}\right) d \tau^{\prime}}\right)\right] .
\end{aligned}
$$

Problematic terms here are the exponentials $\exp \left[i k \int_{\tau_{2}}^{\tau_{1}} \lambda_{j} d \tau^{\prime}\right]$, where $j=1,2$. Let us simplify these terms by introducing an average momentum function

$$
\bar{p}_{j}=\int_{\tau_{1}}^{\tau_{2}} \frac{\lambda_{j}\left(\tau^{\prime}\right) d \tau^{\prime}}{\tau_{2}-\tau_{1}}
$$

Obviously, $\bar{p}_{1}=\bar{p}_{2}=\bar{p}(t)$, where we stressed that the averaged momentum function is a function of time. Changing the integration from times $\left(\tau_{1}, \tau_{2}\right)$ to $\tau=\tau_{1}$ and $s=\tau_{1}-\tau_{2}$, one recasts Eq. (29) in the form

$$
\begin{gathered}
\mathcal{I}\left[\lambda_{1}(\tau), \lambda_{2}(\tau)\right]=-\frac{2 \sigma^{2}}{\tilde{h}^{2}} \int_{0}^{t} d \tau \int_{-t}^{t} d s \int_{-\infty}^{\infty} d k \int_{-\infty}^{\infty} d \omega \\
\times S(k, \omega) e^{i(k \bar{p}-\omega) s}\left[1-\cos \left(k \int_{\tau}^{t}\left(\lambda_{1}-\lambda_{2}\right) d \tau^{\prime}\right)\right] . \quad(31)
\end{gathered}
$$

Integration over $s$ can be approximated by a $\delta$ function. Namely, this integration yields

$$
\int_{-t}^{t} e^{i(k \bar{p}-\omega) s} d s \rightarrow \int_{-\infty}^{\infty} e^{i(k \bar{p}-\omega) s} d s=2 \pi \delta(\omega-k \bar{p}) .
$$

Now integration over the frequency $\omega$ can be performed that yields the action function

$$
\begin{aligned}
& \mathcal{I}\left[\lambda_{1}(\tau), \lambda_{2}(\tau)\right]=\mathcal{I}\left[\lambda_{1}(\tau)-\lambda_{2}(\tau)\right] \\
= & -\frac{2 \sigma^{2}}{\tilde{h}^{2}} \int_{0}^{t} d \tau \int_{-\infty}^{\infty} d k S(k, k \bar{p}) \\
\times & {\left[1-\cos \left(k \int_{\tau}^{t}\left(\lambda_{1}-\lambda_{2}\right) d \tau^{\prime}\right)\right] . }
\end{aligned}
$$

Finally, one obtains

$$
\mathcal{F}\left[\lambda_{1}(\tau), \lambda_{2}(\tau)\right]=\exp \left\{\mathcal{I}\left[\lambda_{1}(\tau)-\lambda_{2}(\tau)\right]\right\},
$$

which is analogous to the expression obtained for Richardson diffusion. Performing again the variable change of Eq. (22), we obtain an expression for the MSD analogous to Eq. (25). The MSD reads

$$
\begin{aligned}
& \left\langle x^{2}(t)\right\rangle=\int_{-\infty}^{\infty} \rho(x, t) x^{2} d x \\
= & \int \frac{d p_{1} d p_{2}}{4 \pi^{2}} \bar{\Psi}_{0}\left(p_{1}\right) \bar{\Psi}_{0}^{*}\left(p_{2}\right) \delta^{(2)}\left(p_{1}-p_{2}\right) \\
\times & \int \prod_{\tau} \frac{d \mu(\tau) d \nu(\tau)}{2 \pi} \exp \left[i \int_{0}^{t} \mu(\tau) \nu(\tau) d \tau\right] \\
\times & \exp \left[-2 i\left(p_{1}-p_{2}\right) \int_{0}^{t} \mu(\tau) d \tau\right. \\
- & \left.\frac{i \tilde{h}}{4}\left(p_{1}+p_{2}\right) \int_{0}^{t} \nu(\tau) d \tau\right] \mathcal{F}[\nu(\tau)] .
\end{aligned}
$$

The essential difference between Eqs. (33) and (25) is the spectral density, which now is a two dimensional function $S(k, k \bar{p})$. Integration over the fields $\mu$ and $\nu$ and differentiation over $p_{1}$ and $p_{2}$ yields

$$
\left\langle x^{2}(t)\right\rangle=\pi \sigma^{2} \int_{0}^{t} d \tau(t-\tau)^{2} \int_{-\infty}^{\infty} S(k, k \bar{p}) k^{2} d k
$$

We obtain the asymptotic behavior of Eq. (34) for large values of $\bar{p}$, following a similar procedure presented in Refs. [2, 3, 34]. Therefore, by rescaling the variables, $k^{\prime}=k \bar{p}$, one obtains

$$
\begin{aligned}
\left\langle x^{2}(t)\right\rangle & =\pi \sigma^{2} \int_{0}^{t} d \tau \frac{(t-\tau)^{2}}{\bar{p}^{3}(\tau)} \int_{-\infty}^{\infty} S\left(\frac{k^{\prime}}{\bar{p}}, k^{\prime}\right) k^{\prime 2} d k^{\prime} \\
& \approx D_{0} \int_{0}^{t} \frac{(t-\tau)^{2}}{\bar{p}^{3}(\tau)} d \tau
\end{aligned}
$$


Here it was reasonable to suppose that $S\left(\frac{k}{\bar{p}}, k\right)$ is a slow function of $k / \bar{p}$. For $\bar{p}=$ const the MSD corresponds to Richardson diffusion $\sim t^{3}$. Such behavior supposes for the averaged momentum function to be an increasing function of time. Moreover, it has been suggested in Ref. [3] that for large $\bar{p}$, one obtains $S(k / \bar{p}, k) \approx S(0, k)$ that yields nonzero generalized diffusion coefficient

$$
D_{0}=\pi \sigma^{2} \int k^{2} S(0, k) d k .
$$

It is also supposes a physical meaning of $\bar{p}^{2}(t)$, which behaves as a velocity-velocity correlation function. Therefore, one suggests a self-consistent procedure, presented in Appendix A, to find this function. This yields for $\bar{p}(t)$

$$
\bar{p}(t)=\left(5 D_{0} / 2\right)^{1 / 5} t^{1 / 5} .
$$

Taking this behavior into account, one obtains

$$
\left\langle x^{2}(t)\right\rangle \sim \bar{D} t^{12 / 5}
$$

which corresponds to hyperdiffusion, observed experimentally [1]. Here $\bar{D}=(2 / 5)^{3 / 5} D_{0}^{2 / 5}$

\section{CONCLUSION}

An enhanced spreading of a quantum wave packet in randomly inhomogeneous media is considered. This quantum process is realized in an arbitrary space-time dependent potential $V(x, t)$. A rigorous quantummechanical calculation of the mean probability amplitude (MPA) is suggested that makes it possible to calculate the mean squared displacement (MSD) of the spreading wave packet. The obtained result establishes the power law spreading of the MSD, which is $\left\langle x^{2}(t)\right\rangle \sim t^{\alpha}$, where $2<\alpha \leq 3$, and the values of the transport exponent $\alpha$ depend on the correlation properties of the random potential $V(x, t)$. The main motivation of the presented analysis is experimental demonstrations on wave packet spreading in random photonic lattices [1, 4, 5]. Another possible application of the presented analysis can be related to a sound waves monitoring in underwater acoustics [22], at the conditions when the parabolic equation approximation is valid and the refractive index has random local fluctuations $\Delta n(x, z)$, which leads to a dominant random potential as in Eqs. (1) and (2).

The rigorous formal expression for the wave function is obtained in a form of paths integration, such that the wave function (9) is a functional of the random potential $V(x, t)$. When $V(x, t)$ is $\delta$ correlated in time as in Eq. (20), the MSD is rigorously calculated in the framework of quantum-mechanical consideration. The dominant term in the MSD of the order of $t^{3}$ is due to turbulent Richardson diffusion [11]. Another important result of Eq (26) is that the quantum homogeneous spread $\sim t^{2}$ stays separate from the dominant classical one $\sim t^{3}$. One can understand this property from the structure of the wave function (9)

$$
\begin{gathered}
\Psi(x, t)=\int D[\lambda(\tau)] \exp \left[\frac{i}{2 \tilde{h}} \int_{0}^{t} d \tau \lambda^{2}(\tau)\right] \\
\times e^{\frac{i}{\tilde{h}} \int_{0}^{t} d \tau V\left(x+\int_{\tau}^{t} d \tau^{\prime} \lambda, \tau\right)} \Psi_{0}\left(x+\int_{0}^{t} d \tau \lambda\right),
\end{gathered}
$$

where $D[\lambda(\tau)]=\prod_{\tau} \frac{d \lambda(\tau)}{\sqrt{2 \pi \tilde{h} i}}$. This is a kind of FeynmanKac formula [20, 21], obtained by means of the auxiliary Markov process ${ }^{3}$ with the Gaussian distribution in the potential $V(x, t)$. However, since $V(x, t)$ is random itself, the details of the potential are not important, and the main information, and contribution to the MPA is due to the correlation function $C\left(x-x^{\prime}, t-t^{\prime}\right)$, or the spectral density $S(k, \omega)$, correspondingly. When the random potential is $\delta$ correlated in time, the auxiliary field $\lambda$ does not intertwine with the potential $V(x, t)$. This is reflected in the solution for the MPA $\rho(x, t)$, where the averaged evolution kernel $\mathcal{F}$ depends only on the quantum part of the auxiliary fields, namely $\mathcal{F}=\mathcal{F}\left[\lambda_{1}(\tau)-\lambda_{2}(\tau)\right]$. As a result of this, rigorous integration over $\lambda_{1}$ and $\lambda_{2}$ is performed. Therefore, each Markov process contributes separately to the MSD in Eq. (26). The quantum mechanics leads to the ballistic $\sim t^{2}$ spread of the initial wave packet, while the classical Obukhov mechanism of turbulent diffusion reveals itself in pure quantum mechanics with the dominant $\sim t^{3}$ spread of the wave packet.

The situation changes dramatically, when the random potential is correlated in both space and time. In this case the auxiliary $\lambda$ fields and the random potential are intertwined due to the nonlocal terms $\int_{t_{1}}^{t_{2}} \lambda(\tau) d \tau$ in the MPA. To make the problem treatable, this nonlocal term is presented in form of an averaged quantum momentum function $\left(t_{1}-t_{2}\right) \bar{p}$, where $\bar{p}$ is related to a velocity-velocity correlation function of random quantum paths. After this approximation, the integration over the $\lambda \mathrm{s}$ is performed rigorously again. Now the quantum ballistic spread is accompanied by hyperdiffusion $\sim t^{\alpha}$. Assuming that the spectral function after rescaling $S\left(\frac{k}{\bar{p}}, k\right)$ is a slow function of $k / \bar{p}$, like in Eq. (36), it is obtained that $\alpha=12 / 5$. As already admitted, this result coincides with one obtained in Refs. 2, 3] in the classical limit of the ray dynamics. However, contrary to Refs. 22, 3], in the present analysis we did not suppose any restriction conditions for the random potential $V(x, t)$.

In the general case, one obtains that $2<\alpha<3$. This result follows from Eq. (35), where $\int_{-\infty}^{\infty} S\left(\frac{k}{\bar{p}}, k\right) k^{2} d k$ is a slow varying function, which approaches to the transport constant $D_{0}$ for the asymptotic large times $t \rightarrow \infty$.

\footnotetext{
${ }^{3}$ Note that quantum mechanics itself is not the Markovian dynamics
} 
Therefore, $\alpha=12 / 5$ is the large time asymptotic result, as well.

\section{Acknowledgments}

I thank Professor S. Fishman for helpful and informative discussions, and comments to the text. This research was supported by the Israel Science Foundation (ISF-1028).

\section{Appendix A: Inferring of the averaged momentum $\bar{p}$}

Let us obtain analytical expression (37) for the averaged momentum $\bar{p}(t)$ in the framework of a self-contained procedure, where we take into account that $\bar{p}^{2}(t)$ is a correlation function. First, it is worth noting that Eq. (30) is a definition of $\bar{p}$. However it does not determine the latter, since the averaging of the random auxiliary field $\lambda(t)$ over the time interval $s=t_{1}-t_{2}$ is not well defined. Second, we admit that the integral $\int_{\tau_{2}}^{\tau_{1}} \lambda(t) d t$ is not zero. Moreover, we replace this integral by a quantum path. One can reasonably suppose that the MSD of these quantum paths is determined by the velocityvelocity correlation function $\bar{p}^{2}(t) \approx\left\langle\dot{x}^{2}(t)\right\rangle_{\lambda}=\left\langle\dot{x}^{2}(t)\right\rangle$. Therefore, we relate the averaged momentum function to the real quantum path $x(t)$, and to estimate its temporal behavior, we consider its classical random dynamics. Integrating the dynamical equation

$$
\ddot{x}=-\frac{d V(x, t)}{d x}
$$

one obtains from the definition of the random potential in Eq. (4)

$$
\dot{x}(t)=\int_{0}^{t} \frac{1}{\sqrt{N}} \sum_{m}(-i) k_{m} A_{m} e^{i k_{m} x\left(t^{\prime}\right)-i \omega_{m} t^{\prime}} d t^{\prime}+c . c .
$$

Therefore, the self-correlation function reads

$$
\begin{aligned}
\left\langle\dot{x}^{2}(t)\right\rangle & =\frac{2 \sigma^{2}}{N} \sum_{m} \int_{0}^{t} d t^{\prime} \int_{0}^{t} d t^{\prime \prime} \\
& \times \cos \left[k_{m}\left(x^{\prime}-x^{\prime \prime}\right)-\omega\left(t^{\prime}-t^{\prime \prime}\right)\right] k_{m}^{2} \\
& =2 \sigma^{2} \int_{0}^{t} d t^{\prime} \int_{0}^{t} d t^{\prime \prime} \int d k \int d \omega k^{2} S(k, \omega) \\
& \times \cos \left[k\left(x^{\prime}-x^{\prime \prime}\right)-\omega\left(t^{\prime}-t^{\prime \prime}\right)\right]
\end{aligned}
$$

Note that according the property (5),$\langle\dot{x}(t)\rangle=0$. After changing integration times $\tau=t^{\prime}$ and $s=t^{\prime}-t^{\prime \prime}$, we arrived at the same expression as in Eq. (31).

Following the solution of the wave function in Eq. (9), the evolution of the coordinates $x(t)$ is due to the shift operator $x(t)=x_{0}+\int_{0}^{t} \lambda(\tau) d \tau$. Therefore, according Eq. (30), the difference $x\left(t^{\prime}\right)-x\left(t^{\prime \prime}\right)$ yields $\bar{p}(\tau)=\left(x^{\prime}-\right.$ $\left.x^{\prime \prime}\right) /\left(t^{\prime}-t^{\prime \prime}\right)=\dot{x}$. Taking into account Eq. (35), one obtains approximately from Eq. (A2)

$$
\bar{p}^{2}(t) \approx D_{0} \int_{0}^{t} \frac{d \tau}{\bar{p}^{3}(\tau)}
$$

Differentiating Eq. A3 over time, one obtains

$$
\bar{p}^{3}(t) \frac{d \bar{p}^{2}(t)}{d t}=D_{0}
$$

This equation can be also obtained by using the well known expression (see e.g., [35])

$$
\left\langle x^{2}(t)\right\rangle_{\lambda}=2 \int_{0}^{t}(t-\tau)\langle\dot{x}(\tau) \dot{x}(0)\rangle_{\lambda} d \tau
$$

and consider that $\left\langle x^{2}(t)\right\rangle=\left\langle x^{2}(t)\right\rangle_{\lambda}$. Now we put forward the physical meaning of the momentum function $\bar{p}$ by substituting it in Eq. A5

$$
\left\langle x^{2}(t)\right\rangle_{\lambda}=2 \int_{0}^{t}(t-\tau) \bar{p}^{2}(\tau) d \tau
$$

Differentiating twice Eqs. (A6) and (35) over $t$ and comparing the obtained results, one obtains

$$
\bar{p}^{2} \approx D_{0} \int_{0}^{t} \frac{d \tau}{\bar{p}^{3}(\tau)}
$$

Differentiating this over time again, one obtains Eq. (A4). Solving this equation, one obtains

$$
\bar{p}(t)=\left(5 D_{0} / 2\right)^{1 / 5} t^{1 / 5}
$$

[1] L. Levi, Y. Krivolapov, S. Fishman and M. Segev, Nature Phys. 8, 912 (2012).

[2] E. Arvedson, M. Wilkinson, B. Mehlig, and K. Nakamura, Phys. Rev. Lett. 96, 030601 (2006).

[3] Y. Krivolapov, L. Levi, S. Fishman, M. Segev and M.
Wilkinson, New J. Phys. 14, 043047 (2012).

[4] ) L. Levi, M. Rechtsman, B. Freedman, T. Schwartz, O. Manela and M. Segev, Science 332, 1541 (2011).

[5] M. Rechtsman, L. Levi, B. Freedman, T. Schwartz, O. Manela and M. Segev, Optics and Photonics News (Spe- 
cial Issue: Optics in 2011) $\mathbf{2 2}$ (12), (2011).

[6] V.I. Kliatskin, Stochastic equations and waves in randomly inhomogeneous media (Nauka, Moscow, 1980) (in Russian).

[7] R. Dashen J. Math. Phys. 20894 (1979); Opt. Lett. 9, 110 (1984).

[8] J. Zinn-Justin, Quantum Field Theory and Critical Phenomena (Claredon Press, Oxford, 1990).

[9] V.I. Kliatskin, Phys. Uspekhi 47, 169 (2004).

[10] E. D. Tappert, The Parabolic Approximation Method, Lectures Notes in Physics, 70, in: Wave Propagation and Underwater Acoustics, eds. by J. B. Keller and J. S. Papadakis, (Springer, New York, 224-287, 1977).

[11] L. F. Richardson, Proc. R. Soc. A 110, 709 (1926).

[12] U. Frisch, Turbulence. The Legacy of Kolmogorov (Cambridge Univ. Press, Cambridge, 1995).

[13] A.S. Monin, A.M. Yaglom, Statistical Fluid Mechanics: Mechanics of Turbulence vol. 1 ( MIT Press, Cambridge, 1971); ibid vol 2 (MIT Press, Cambridge, 1975).

[14] A.M. Obukhov, Adv. Geophys. 6, 113 (1959).

[15] A.M. Jayannavar and N. Kumar, Phys. Rev. Lett. 48, 553 (1982).

[16] K. Furutsu, J. Res. N.B.S. D67, 303 (1963).

[17] E.A. Novikov Zh. Eksp. Teor. Fiz. 47, 1919 (1964) [Sov. Phys. JETP 20, 1990 (1965)].

[18] D.A. Kessler and E. Barkai, Phys. Rev. Lett. 108, 230602 (2012).

[19] Y. Sagi, M. Brook, I. Almog, and N. Davidson, Phys. Rev. Lett. 108, 093002 (2012).
[20] R.P. Feynman and A.R. Hibbs, Quantum Mechanics and Path Integrals (McGrow Hill, New York, 1965).

[21] M. Kac, Probability and Related Topics in Physical Sciences. (Interscience, New York, 1958).

[22] A.L. Virovlyansky, D.V. Makarov, and S.V. Prants, Phys. Uspekhi 55, 18 (2012).

[23] K. C. Hegewisch and S. Tomsovic Europhys. Lett. 97, 34002 (2012).

[24] M.A. Leontovich, Izv. USSR Ac.Sc., Phys. 8, 16, 1944 (in Russian).

[25] R.V. Khohlov, Radiotech. and Elrctron. 6, 1116, 1961 (in Russian).

[26] R.L. Stratonovich, Dokl. Akad. Nauk SSSR 115, 1097 (1957) [Sov. Phys. Dokl. 2, 416 (1958)].

[27] J. Hubbard, Phys. Rev. Lett. 3, 77 (1958).

[28] V.V. Sokolov, Theor. Math. Phys. 61, 1041 (1984).

[29] G.P. Berman and A.M. Iomin, Theor. Math. Phys. 77, 1197 (1988).

[30] A. Iomin, Phys. Rev. E 70, 026206 (2004).

[31] P. Carruthers and M.M. Nieto, Rev. Mod. Phys. 40, 411 (1968).

[32] G. Falkovich, K. Gawedzki, and M. Vergassola, Rev. Mod. Phys. 73, 913 (2001).

[33] A. Baule and R. Friedrich, Phys. Lett. A 350, 167 (2006).

[34] V. Bezuglyy, B. Mehlig, M. Wilkinson, K. Nakamura, and E. Arvedson, J. Math. Phys. 47, 073301 (2006).

[35] F. Reif, Fundamentals of statistical and thermal physics (McGraw Hills, New York, 1965). 